\title{
Protein Arrays as Tools for Detection of Protein-Protein Interactions by Mass Spectrometry
}

\author{
Christian F. W. Becker ${ }^{1}$, Ron Wacker ${ }^{2}$, Werner Bouschen ${ }^{3}$, Ralf P. \\ Seidel $^{1}$, Branko Kolaric ${ }^{1}$, Pascal Lang ${ }^{1}$, Hendrik Schroeder ${ }^{2,4}$, Christof \\ Niemeyer ${ }^{4}$, Bernhard Spengler ${ }^{3}$, Roger S. Goody ${ }^{1}$ and Martin \\ Engelhard $^{1}$
}

${ }^{1}$ Max-Planck-Institut für molekulare Physiologie, Otto-Hahn-Str. 11, 44227 Dortmund; ${ }^{2}$ Chimera Biotech GmbH, Emil-Figge-Str. 76 A, 44227 Dortmund; ${ }^{3}$ Justus-Liebig Universität Giessen, Schubertstr. 60, 35392 Giessen; ${ }^{4}$ Universität Dortmund, Otto-Hahn-Str. 6, 44227

Dortmund, Germany

\section{Introduction}

DNA-Protein arrays can be developed into tools for the detection of protein-protein interactions and identification of protein binding partners by mass spectrometry. An important advantage of mass spectrometry readout of microrarrays is the molecular weight information that is obtained for the analyte. Such a readout system has been successfully used in approaches to determine protein expression levels, to follow enzyme activity, and to observe proteins bound to immobilized ligands [1-4]. Here we describe a microarray that combines the reversible immobilization of proteins with readout by MALDI mass spectrometry in order to study protein-protein interactions and to identify binding partners of immobilized proteins. The immobilization strategy is based on the much further advanced technology of DNA chips, utilizing protein-DNA conjugates to achieve highly specific but non-covalent binding to the surface-attached oligonucleotides [5-7].

The Ras protein and the Ras-binding domain of its effector protein cRaf-1 were chosen as a test system for this approach [8]. The Ras protein plays a vital role in the transduction of extracellular signals from the cell membrane towards the nucleus by cycling between its activated, GTP-bound state in which it interacts with its downstream effectors and its inactive GDP-bound state [9]. Mutations in the Ras protein can lead to constitutive activation of signaling cascades either by deactivation of GTP hydrolysis or by preventing the interaction with Ras GAPs. Such mutations occur in $30 \%$ of all solid human cancers [10]. These facts turn the Ras-RBD protein pair into a highly interesting test case where two isoforms of a protein (Ras) with different affinities for the capture protein (RBD) have to be distinguished. A potential diagnostic use can also be deduced.

\section{Results and Discussion}

In order to achieve functional immobilization of the protein samples in a controlled manner, protein-DNA fusion proteins were synthesized. The synthesis strategy is based on the expressed protein ligation approach. A protein with a C-terminal thioester and an oligonucleotide that carries a 5 '-cysteine modification are linked by an amide bond (Fig. 1A) [11,12]. The protein thioesters were generated from recombinantly produced intein fusion proteins that, upon treatment with mercaptoethanesulfonic acid (Mesna), released Ras- and RBD-thioesters. The cysteine-modified oligonucleotide was synthesized using standard phosphoramidite chemistry and a cysteine-modified thymidine building block. 


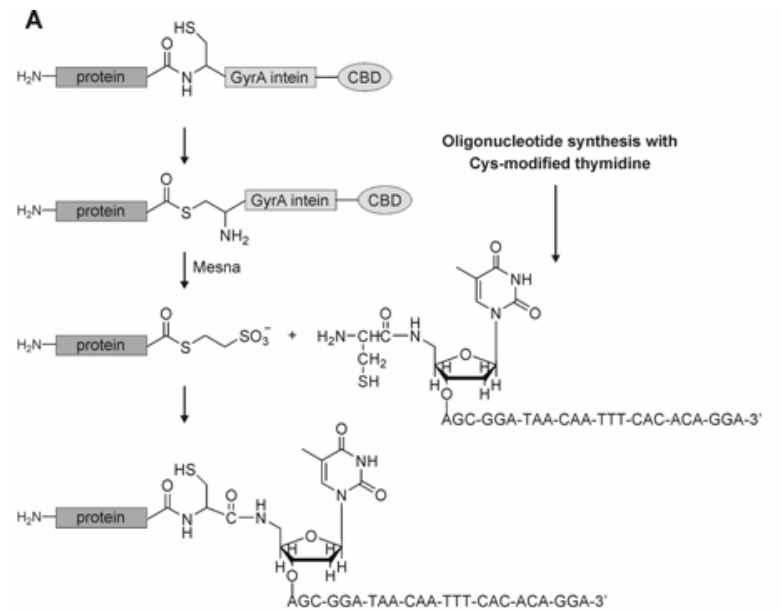

B

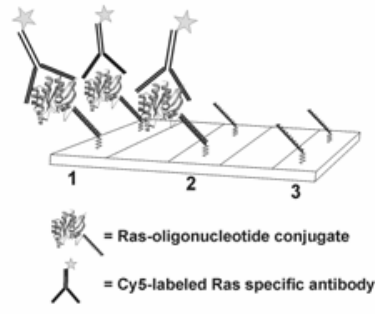

C

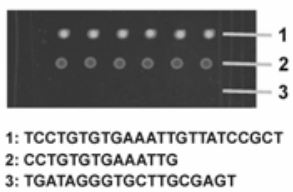

Fig. 1. A) Schematic representation of the ligation reaction of $R B D$ and Ras thioesters with 5'-Cys-modified oligonucleotide to produce the capture proteins RBD-DNA and Ras-DNA. B) Schematic representation of Ras-DNA detection on glass arrays with a fluorescently labeled Ras-specific antibody. C) Fluorescence readout signal of Ras-DNA-antibody complex.

The immobilization strategy was initially tested with Ras- and RBD-DNA conjugates on glass microarrays using conventional fluorescence readout (Fig. 1B). The glass surface was covalently modified with commercially available 5'-amino modified oligonucleotides. Immobilized Ras-DNA was detected after incubation with a Cy5-labeled Ras-specific antibody. Specific interaction between a fully complementary surface-attached oligonucleotide $\mathbf{1}$ and the Ras-DNA conjugate was clearly demonstrated by the strong fluorescence signal (Fig. 1C). A complementary but truncated oligonucleotide $\mathbf{2}$ on the surface produced a much weaker signal indicating a lower amount of bound Ras-DNA. A non-complementary oligonucleotide 3 did not produce any signal.

This DNA-directed immobilization (DDI)-based approach was successfully transferred towards silica-based microarrays that were combined with a MALDI readout system. The interaction of the Ras-RBD protein pair was unambiguously detected by using RBD-DNA conjugates as capture molecules on the microarrays and activated Ras:GppNHp (GppNHp is a non-hydrolyzable GTP analog) as the analyte. The incubation with analyte protein was followed by several washing steps and application of a layer of organic matrix (sinapinic acid) in order to allow efficient ionization before insertion into the home-build MALDI spectrometer (Fig. 2A) [13]. Multiple control experiments, e.g., with inactive Ras:GDP, did not produce a MALDI signal (Fig. 2B). However, after incubation with activated Ras, subsequent washing and matrix application, the Ras protein (calculated MW 18853 Da) was easily detected in the mass spectrum (Fig. 2C).

In conclusion, it was shown that MALDI mass spectrometry is a suitable detection method for DDI-based protein microarrays. The presented approach provides highly selective immobilization of capture and analyte molecules as demonstrated for the interacting protein pair Ras and RBD. 

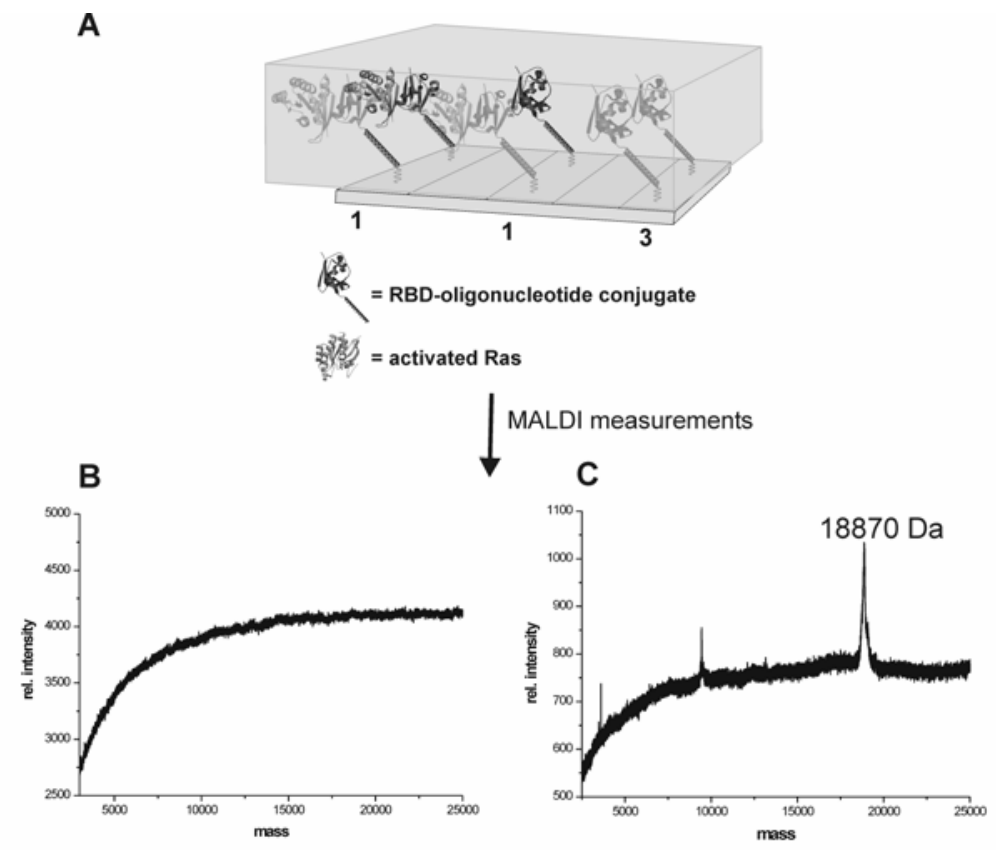

Fig. 2. A) Ras captured by immobilized RBD-DNA on a silica-based array covered with a layer of organic matrix. B) Typical readout signal in control experiments. C) Typical readout after incubation of immobilized RBD-DNA with activated Ras:GppNHp.

\section{Acknowledgement}

This work was funded in part by the MPG, DFG, BMBF and VCI.

\section{References}

1. Seibert, V., Wiesner, A., Buschmann, T. and Meuer, J. Pathol. Res. Pract. 200, 83-94 (2004).

2. Min, D. H., Su, J. and Mrksich, M. Angew. Chem. Int. Ed. Engl. 43, 5973-5977 (2004).

3. Min D. H. and Mrksich, M. Curr. Opin. Chem. Biol. 8, 554-558 (2004).

4. Yeo, W. S., Min, D. H. R., Hsieh, W., Greene, G. L. and Mrksich, M. Angew. Chem. Int. Ed. Engl. 44, 5480-5483 (2005).

5. Niemeyer, C. M. Biochem. Soc. Trans. 32, 51-53 (2004).

6. Lovrinovic, M., Seidel, R., Wacker, R., Schroeder, H., Seitz, O., Engelhard, M., Goody, R.

S. and Niemeyer, C. M. Chem. Commun. 822-823 (2003).

7. Wacker, R., Schroder, H. and Niemeyer, C. M. Anal. Biochem. 330, 281-287 (2004).

8. Wittinghofer A. and Waldmann, H. Angew. Chem. Int. Ed. Engl. 39, 4193-4214 (2000).

9. Boguski M. S. and McCormick, F. Nature 366, 643-654 (1993).

10. Barbacid, M. Annu. Rev. Biochem. 56, 779-827 (1987).

11. Muir T. W. Annu. Rev. Biochem. 72, 249-289 (2003).

12. Takeda, S., Tsukiji, S. and Nagamune, T. Bioorg. Med. Chem. Lett. 14, 2407-2410 (2004).

13. Spengler, B. J. Mass Spectrometry 32, 1019-1036 (1997). 